\title{
Neural-Based Control of A Robotic Hand: Evidence for Distinct Muscle Strategies
}

\author{
Pedram Afshar*§, Yoky Matsuoka†§ \\ * BioMedical Engineering, $\uparrow$ The Robotics Institute \& Mechanical Engineering \\ $\S$ Center for the Neural Basis of Cognition \\ Carnegie Mellon University, Pittsburgh, Pennsylvania, 15213 \\ Email: pedram@cmu.edu, yoky@andrew.cmu.edu
}

\begin{abstract}
The neural-based control of a robotic hand has many clinical and engineering applications. Current approaches to this problem have been limited due to a lack of understanding of the relationship between neural signals and dynamic finger movements. Here, we present a technique to predict index finger joint angles from neural signals recorded from the associated muscles. The neural signals are converted to a torque estimate (EBTE) and then input to artificial neural networks. The networks predict the finger position more closely when the input to the networks are torque estimates rather than neural signals. Furthermore, the networks trained with the EBTE signals could predict the joint angles for different phases of finger movements (i.e. dynamic reaching and positioning task) while networks trained with the neural signals could not. Our results indicate that (1) similar finger movements are executed with different synergistic strategies and (2) different phases of finger movements employ different neural strategies. Through these results, we have demonstrated the first concrete technique to control a hand prosthetic device or dexterous tele-manipulator using natural neural control signals.
\end{abstract}

Keywords-motor control, electromyography, joint angles

\section{INTRODUCTION}

Discovering the relationship between neural signals and limb position would enable the development of natural brainmachine interfaces. Tele-operated devices controlled by neural commands could be used to provide precise human manipulation in remote or hazardous environments, and neurally controlled prosthetics could return function to a paralyzed patient by routing neural commands to actuators [1]. These applications rest on the assumption that neural activity can be translated into intended movements, and that these movements can then be executed by a device [1-3].

Decoding the neural signal into movement parameters (i.e, position and velocity) continues to be the major hurdle in solving this problem. Since Georgopolous et al [4] predicted gross arm movement direction from neural activity in monkey motor cortex, many others have used a variety of top-down approaches (central nervous system to movement) to predict dynamic movement direction $\left[\begin{array}{ll}5,6 & 6\end{array}\right.$. Georgopolous et al [4] found that neurons fired selectively for a particular direction of hand movement, following a roughly cosine tuning curve with respect to movement direction. Schwartz et al [5] further showed that averaging these movement vectors over a group of motor neurons correlated with arm movement direction. To establish a more concrete relationship between neurons and limb position, Fetz et al [7] [2] has shown that neural activity downstream of the brain (in the spinal cord) can be used to predict muscle activity. Work by Holdefer et al [8] extended these results by showing that the activity of single neurons in the monkey brain caused activation in specific sets of limb muscles.

This type of neural signal processing has enabled the development of brain-machine interfaces (BMI), which control robots using measured neural activity in rats and monkeys $[1,15,16]$. These devices work by correlating sampled neuronal activity in the brain and with limb movement direction through various classification techniques. That is, these algorithms use the neural signal to select one arm direction from a set of possible directions rather than computing the continuous limb movement. While these top-down approaches hold promise for the construction of a neuroprosthetic device, they can only provide coarse estimation of the limb position. This is because it is not clear what the neural signal is encoding. In producing a movement, the neural signal is modified in the brain, the spinal cord, and by the muscle itself.

The method of predicting dynamic and fine movements directly from cortical signals treats the brain, spinal cord, and muscle as a black box. To predict fine and dynamic movements, it is necessary to take a bottom-up approach. The first step is to understand the relationship between the neural signal to muscle and the movement itself. This mapping, however, is not trivial because the neural signals to the muscles are known to have a nonlinear and many-to-one relationship with the forces that muscles produce. Furthermore, the complex biomechanical mapping between the muscle forces and the joint angles make the already many-to-one redundant relationship a difficult mapping to model.

In this paper, we present the first successful method for determining precise finger position of a human in a dynamic movement task using neural input to muscles. While subjects traced a line with their index finger, we collected neural signals from all seven muscles that control the index finger. We describe a technique to convert the neural input into a quantity that resembles joint torques as a way to 
resolve the redundant relationship. Then we describe an artificial neural network that takes this quantity as its input and predicts the joint angles accurately. Using this network, we hypothesize that the neural network trained with the torque-like quantity can predict joint angles for all conditions, while the network trained with the unprocessed neural signals can only predict specific phases of a movement.

\section{METHODS}

\section{A. Experimental Setup}

The neural input to the muscles and the joint angles were measured from two subjects ( 2 male, age 21,23$)$ while they made a point-to-point movement with their right index finger (Fig. 1). We used a PHANToM ${ }^{\mathrm{TM}}$ Premium 1.5 robot (Sensable Technologies, Inc.) to record the index finger movement. This robot has 3 degrees of freedom and a custom-made finger cuff provided an additional two rotational degrees of freedom. The PHANToM recorded the Cartesian position of the robot endpoint, and we attached a potentiometer to the pitch axis of the cuff to record the pitch of the fingertip. The subjects' index finger was fastened into the finger cuff so that their distal interphalangeal joint (DIP) was aligned to the edge of the cuff. The Cartesian position of the DIP was determined by calculating its geometric relationship with the PHANToM endpoint. The subjects' palm and other digits were strapped to an armrest so that all the joints on the index finger could move freely while the hand was fixed a known distance away from the robot's origin. We recorded the joint angles of the robot and the finger cuff at $1 \mathrm{kHz}$.

Given that the hand is fixed and the robot is rigidly coupled to the fingertip, inverse kinematics equations can uniquely identify all four joint angles, provided the correct range of motion for the joints. Assuming that $\left(P w_{x}, P w_{y}, P w_{z}\right)$ is the location of the distal interphalangeal joint (DIP) center ,$\Phi$ is the angle measured by the finger cuff, $\mathrm{a}_{2}$ is the distance from the metacarpophalangeal joint (MCP) to proximal interphalangeal joint (PIP) and $\mathrm{a}_{3}$ is the distance from the PIP to the DIP. The MCP flexion is determined by

$$
M C P=A \tan 2\left(\frac{\left(a_{2}+a_{3} c_{3}\right) P w z-\sqrt{P^{2} w_{y}+P^{2} w_{x}}}{P^{2} w_{x}+P^{2} w_{y}+P^{2} w_{z}}, \frac{\left(a_{2}+a_{3} c_{3}\right) \sqrt{P^{2} w_{y}+P^{2} w_{x}}+a_{3} s_{3} P w_{z}}{P^{2} w_{x}+P^{2} w_{y}+P^{2} w_{z}}\right)
$$

the MCP abduction is determined by

$$
A B D=A \tan 2\left(P w_{y}, P w_{x}\right),
$$

the PIP flexion is determined by

$$
P I P=A \tan 2\left\{\sqrt{1-\left(\frac{P^{2} w_{x}+P^{2} w_{y}+P^{2} w_{z}-a_{2}{ }^{2}-a_{3}{ }^{2}}{2 a_{2} a_{3}}\right)^{2}}, \frac{P^{2} w_{x}+P^{2} w_{y}+P^{2} w_{z}-a_{2}{ }^{2}-a_{3}{ }^{2}}{2 a_{2} a_{3}}\right\}
$$

and the DIP flexion is determined by

$$
D I P=\Phi-(P I P+M C P) .
$$

Along with the index finger joint angle information, neural input to all the muscles controlling the index finger

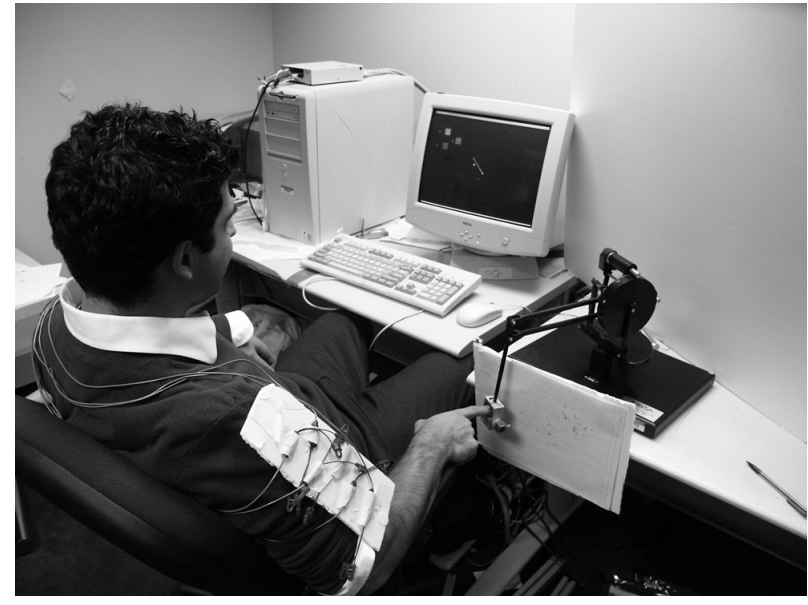

Figure 1. Experimental setup. The subject's index fingertip endpoint and the neural inputs to the muscles controlling the index finger were measured as the subject traced a line with his index fingertip.

were measured: Flexor Digitorum Superficialis, Flexor Digitorum Profundus, Extensor Indicis, Extensor Digitorum, First Lumbrical, First Dorsal Interosseus, and First Palmar Interosseous. We used fine-wire electromyographic (EMG) technique modified from Burgar et al [9, 10]. A $30 \mathrm{~cm}$ segment of $50 \mu \mathrm{m}$ bifilar wire (California Fine Wire) was threaded through a 27 gage hypodermic needle. Muscles were identified using anatomical landmarks and signals were tested for cross-talk by viewing the signal on an oscilloscope prior to recording. At the beginning and end of the session, the maximum and minimum muscle contraction signals were recorded. To find the minimum, the subjects placed their hand on the table and were asked to relax and let the table support their arm and hand. The maximum contraction signal was determined for each muscle individually by asking the subject to contract as hard as possible against the table in the direction of the muscle's action. During the experiment, the subjects were instructed to trace a $3.5 \mathrm{~cm}$ line segment with a tracing error of less than $1 \mathrm{~mm}^{2}$ and in a time window between 450 and 550 milliseconds. The tip of the index finger traveled from the upper left to the lower right corner of the index finger workspace. This motion was selected because it spanned a wide range of each of the finger joint angles. At the end of each trial, the subject was given performance feedback on a computer monitor. The subject's trace was superimposed on a drawing of the line segment, and colored boxes indicated the outcome in achieving the desired time window and tracing error performance. After observing the error, the subject reset his finger to the starting point and waited for an audible go stimulus to begin the next trace.

The session lasted for 120 movements. In order to assure that all movements used for the analysis were similar, we excluded the first 60 movements (to exclude movements that may include any adaptation changes) from the analysis. We also stopped recording at 120 movements to prevent fatigue, which may change the nature of the EMG signal [11]. 


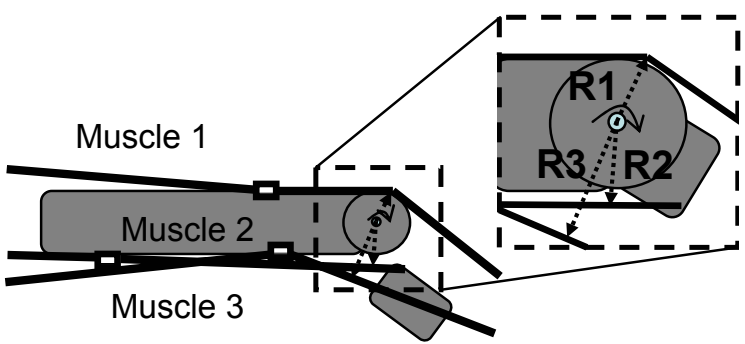

Figure 2. Model of cocontraction in a joint of the index finger. Joint movements are determined by torque around the joint.

\section{B. Data analysis}

For each movement, there were 7 EMG recordings and 4 joint angle measurements for a period of 250-750 milliseconds, depending on the time it took for the subject to reach the endpoint. The goal of the analysis was to design a mapping from EMG to joint angles as a function of time. We created two different sets of inputs for the artificial neural network to predict the joint angles.

\section{1) Normalized EMG}

EMG signals are a composite of the neural signals from many motor-neurons. As such, the signal is irregular, chaotic, and oscillatory. However, the amount of motorneuron activity is correlated with the number of zerocrossings, the number of times the signal crosses 0 volts[10]. Therefore, the amount of muscle force, which is a result of motor-neuron firing, could be hypothesized to be proportional to the frequency of zero-crossings.

Furthermore, an estimate of the force of contraction can be obtained by normalizing the zero-crossings frequency using the maximum and minimum contraction. The magnitude of the EMG signal was determined by taking the number of zero-crossings in a 15-millisecond time window. The signal was normalized and low pass filtered at $70 \mathrm{~Hz}$ with an equiripple FIR filter with Kaiser window. This filtered and normalized EMG was one of the inputs used to estimate the joint angles.

\section{2) EMG Based Torque Estimate}

To produce the second set of inputs, we used the assumption that this filtered and normalized EMG signal is proportional to muscle force [12] to calculate a quantity that is related to the joint torque. Our EMG Based Torque Estimate (EBTE), is determined by combining the EMG signals of muscles around a joint (Fig. 2). For each joint, an EBTE was computed by

$$
\hat{\tau}_{j}(t)=\sum_{m} \hat{F}_{m}(t) \cdot r_{m, j}
$$

where $\hat{F}_{m}(t)$ is the force estimate from the EMG and $r_{m, j}$ is the radius of the moment arm for each muscle around the joint [13] (Fig. 2). Using seven EMG signals, we calculated four EBTEs corresponding to each degree of freedom.
Since there are more muscles than degrees of freedom, there are infinitely many muscle contraction patterns that impart the same torque on a joint in the index finger. These patterns represent differences in cocontraction (simultaneous activity of opposing muscles around a joint), which changes joint stiffness. Computing the EBTE effectively removes the redundant muscle actuation to joint angle mappings. While the EBTE resembles the joint torques, literature suggests that EMG signals are not equivalent to muscle force [10, 14]. Thus, the EBTE, which uses $\hat{F}_{m}(t)$, cannot be considered as the joint torque signal, and thus cannot be used in the traditional dynamical equations of motion.

Instead, a two layer artificial neural network was used to predict joint angles from the normalized EMG and the EBTE. The network, implemented using MATLAB, consisted of a single input layer and a single output layer. The input layer had 4 (EBTE network) or 7(EMG network) nodes, each connected to a 40 millisecond tapped delay line. The output layer consisted of 4 nodes, and the hidden layer consisted of 15 nodes. The network was trained with scaled conjugate gradient descent backpropagation with 10 input sets until achieving a mean-squared-error of 0.05 .

All movements to the target were stereotypical reaching movements in that they were performed in two distinctive phases shown in Fig 3. The first phase, called the "reaching phase," contained a high velocity (greater than $80 \mathrm{~mm} / \mathrm{sec}$ ) profile towards the endpoint. The second phase, called the "corrective" phase, contained a slower velocity profile (between 20 and $70 \mathrm{~mm} / \mathrm{sec}$ ) to correct the error of the reaching phase and position the fingertip at the endpoint. Because we hypothesized that the reaching and corrective phases may use different mappings between the muscles and the joint angles, we treated those two phases separately while training the neural network. Rather than dividing the whole movement into two segments, we took the reaching phase to be the segment where the first bell-shaped profile exceeded $75 \mathrm{~mm} / \mathrm{sec}$, and the corrective phase to be the segment where the second bell-shaped profile exceeded $20 \mathrm{~mm} / \mathrm{sec}$. This segmentation technique allowed multiple reaching movements to align properly during the network training. We also tested a "whole" movement condition. The whole movement was taken from the beginning of the reaching phase until the end of the corrective phase.

For each training session, 13 movements were selected at random from the 60 total movements. The network was trained with 10 movements selected at random from the selected 13 movements. After reaching the convergence criterion (MSE < 0.05), the network was tested on the 3 input sets it had not been given. Joint angles calculated from the neural network were compared with joint angles determined from the inverse kinematics equations (equations (1) - (4)).

Using the data collected, the EMG network and EBTE network was compared in the following ways:

- Predicting joint angles for the whole movement. If the redundancy is well represented in the EBTE model, the network trained with the EBTE should 
A

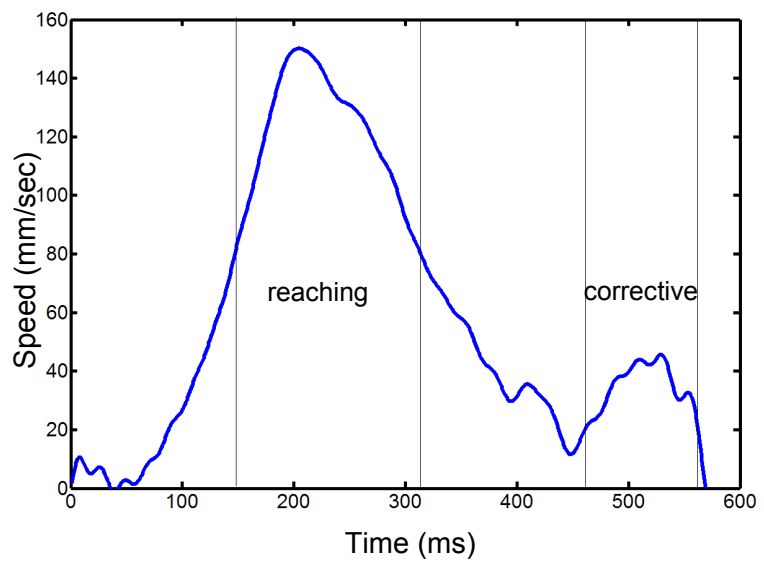

B

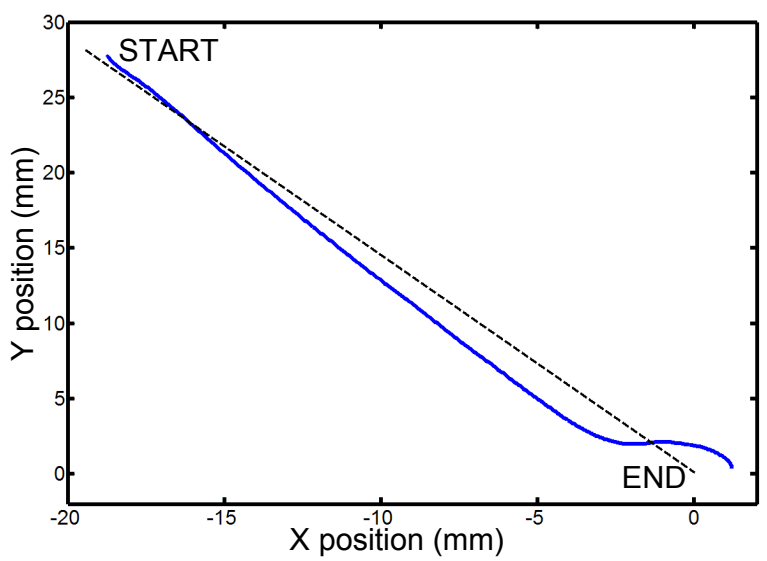

Figure 3. Line tracing task. A: The speed profile of the trace as a function of time shows two bell-shaped curves: the reaching movement $(150 \mathrm{~ms}-310 \mathrm{~ms})$ and the corrective movement $(475 \mathrm{~ms}-550 \mathrm{~ms})$. B: The position of the index fingertip during the trace. The dotted line indicates the prompt, the solid line indicates the subject's trace.

predict the joint angles more accurately than the normalized EMG.

- $\quad$ Predicting joint angles for the reaching phase based on networks trained on only the reaching phase. Both networks should also be able predict the reaching phase since cocontraction strategy is constant (see discussion).

- $\quad$ Predicting joint angles for corrective phase based on the networks trained on the reaching phase. Since only the EBTE network should have a unique mapping to the joint movement, it should predict joint angles for reaching or corrective phases even if the network was trained on the reaching phase only. If the cocontraction strategies are different between these two phases, then the EMG network should predict joint angles poorly in this condition.

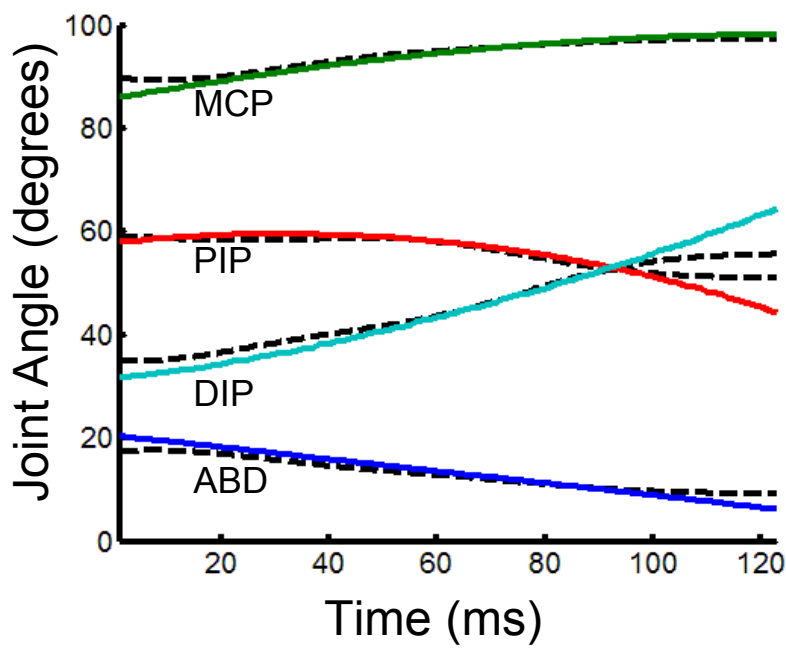

Figure 4. Joint angle predictions of a neural network based on EBTE. MCP = metacarpophalangeal joint flexion, $\mathbf{A B D}=$ metacarpophalangeal joint abduction, DIP = distal interphalangeal joint PIP = proximal interphalangeal joint.

\section{RESULTS}

\section{A. Predicting Whole Movements with a Network Trained on Whole Movements}

When the input to neural network was the EBTE of the whole movement, it converged after 90 epochs. The comparison between the actual and predicted movements for a test movement (not used during training) is shown in Fig. 4. For 3 testing movements, the average $\mathrm{R}^{2}$ was $0.76 \pm 0.14$. When the normalized EMG was used as the input, it converged after 85 epochs and predicted the testing movements with an average $\mathrm{R}^{2}$ of $0.70 \pm 0.16$. Though we predicted better performance from the EBTE network, the improved performance was not statistically significant $(\mathrm{p}<0.61)$.

\section{B. Predicting Reaching Movements with a Network Trained on Reaching Movements}

When the input to the neural network was the EBTE of the reaching movement only, it converged after 70 epochs. For the 3 testing movements, the average $\mathrm{R}^{2}$ was $0.83 \pm 0.09$. When normalized EMG was used to predict reaching movements, the network converged in 55 trials with an average $\mathrm{R}^{2}$ of $0.81 \pm 0.05$ for the testing movements (Fig 5). As expected, both networks were able to predict joint angles well.

\section{Predicting Corrective Movements with a Network Trained on Reaching Movements}

Finally, the network trained on the reaching movements alone was used to predict corrective movements. The EBTE network predicted corrective phase joint angles with an average $\mathrm{R}^{2}$ of $0.84 \pm 0.16$. When normalized EMG was used as input, the network predicted corrective movement joint angles with an average $\mathrm{R}^{2}$ of $0.58 \pm 0.14$ (Fig 5). 


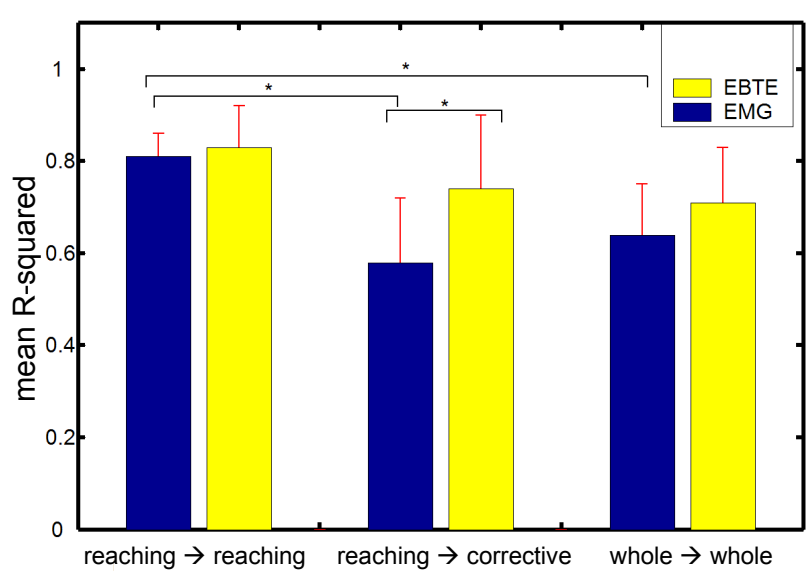

Figure 5. Performance of EBTE netowork versus EMG network under three testing conditions. The performance of the EBTE network was not statistically different in any of the testing conditions: 1) trained on reaching phase, tested on reaching phase 2 ) trained on reaching phase, tested on corrective phase, 3 ) trained on whole trial and tested on whole trial. The performance of the EMG network was statistically poorer $(p<0.05)$ in conditions 2 and 3 than on condition 1. Furthermore, the EBTE network performed statistically better than the normalized EMG network in condition 2.

\section{DISCUSSION}

The control of neuroprosthetics rests heavily on the ability to use neural signals to predict limb position. In the past, neural signals have been used to predict gross direction and muscle activation patterns in the hand and arm [1-8]. Though this work has been used to grossly control a prosthetic device, it lacks the fine resolution necessary to make a fully functional neural prosthetic for humans. To achieve this goal, one of the necessary hurdles to overcome is the continuous mapping between the neural input to muscles and the position of a limb. To date, there have not been published reports demonstrating this mapping.

In our results, we have shown that both EBTE and normalized EMG predicted dynamic index finger movements well (mean $\mathrm{R}^{2}=0.78$ ). Both networks were also able to predict dynmamic movements (mean $\mathrm{R}^{2}=0.82$ ) in the reaching phase alone. Nevertheless, we hypothesized that EBTE would be a more robust predictor of position since, unlike EMG, it was designed to be insensitive to varying cocontraction strategies.

To test this hypothesis, we trained the networks on the reaching phase and compared their performance on predicted reaching and corrective phase movement. It has been shown that increasing the positional accuracy of a movement increases the level muscle cocontraction[17]. Since greater positional accuracy is required during the corrective movement than the reaching movement, it is likely that different cocontraction strategies are utilized to execute the two movements. Therefore, we hypothesized that only the EBTE trained network would be able to predict both the reaching and corrective movements. The EMG trained network would be able to predict finger position only in the phase in which it was trained. Fig 5 shows a statistically significant $(\mathrm{p}<0.05)$ decrease in performance when the EMG network was tested on the corrective phase movement. No statistical difference was found between the EBTE network's prediction of reaching movements and corrective movements. This result shows that 1) the EBTE we calculated is a good model to eliminate the cocontraction effect, and 2) using the EBTE, the joint angles could be reliably predicted regardless of the neural strategies used.

Given our hypothesis that the EBTE is a better predictor of limb position than EMG, it is surprising that the EMG trained network performed statistically the same as the EBTE trained network on the whole trial data. One explanation is that the reaching movement was overly represented in the whole trial data. In fact, the reaching movement phase composed approximately $3 / 4$ of the whole trial. Thus, it is possible that the EMG trained network learned only the cocontraction pattern found in the reaching phase and could therefore accurately predict the joint angles in a large portion of the whole trial. The total performance of the normalized EMG network would therefore be inflated. This hypothesis is supported by the fact that the normalized EMG network is qualitatively better at predicting the joint angles in the first 210 milliseconds of the trial (Fig 4b). This potentially spurious result could be resolved by selecting movement phases of equal duration.

We have demonstrated the first mapping between neural input to muscles and their associated joint angles in a dynamic movement task. There are two logical next steps. The first is to extend the network to include other fingers in the hand. By monitoring joint position and muscle activity, the predictive ability of the network can be extending to capture whole hand movements. The second is to use the neural signals found in the higher neural structures like the spinal cord in order to predict the neural input to the muscles. In this way, we would produce a mapping from neural signals originating in the spine to limb position. Together, these advances will allow us to develop a brain-machine interface that decodes neural signals to produce a fullyfunctional neuroprosthetic hand.

\section{REFERENCES}

[1] Chapin, J., et al., Real-time control of a robot arm using simultaneously recorded neurons in the motor cortex. Nature neuroscience, 1999. 2: p. 664-670.

[2] Fetz, E.E., et al., Roles of primate spial interneurons in preparation and execution of voluntary hand movement. Brain Research Reviews, 2002. 40: p. 53-65.

[3] Fetz, E.E.S., L.E.; Murthy, V.N. Neural networks controlling wrist movements. in Neural Networks, 1990., 1990 IJCNN International Joint Conference on. 1990.

[4] Georgopoulos, A., et al., On the relations between the direction of two-dimensional arm movements and cell discharge in primate motor cortex. Journal of Neuroscience, 1982. 2: p. 1527-1537.

[5] Moran, D. and A. Schwartz, Motor cortical representation of speed and direction during reaching. Journal of Neurophysiology, 1999. 82: p. $2676-2692$ 
[6] Taylor, D., S.H. Tillery, and A. Schwartz, Direct Cortical Control of $3 D$ Neuroprosthetic Devices. Science, 2002. 296: p. 1829-1832.

[7] Fetz, E.E., S.I. Perlmutter, and Y. Prut, Functions of mammalian spinal interneurons during movement. Current opinion in Neurobiology, 2000. 10: p. 699-707.

[8] Holdefer, R.N. and L.E. Miller, Primary motor cortical neurons encode functional muscle synergies. Experimental Brain Research, 2002. 146: p. 233-243.

[9] Burgar, C., F.J. Valero-Cuevas, and V. Hentz, Fine-Wire electromyographic recording during force generation: Application to Index Finger Kinesiologic Studies. American Journal of Physical Medicine and Rehabilitation, 1997. 76(6): p. 494- 501.

[10] Loeb, G.E. and C. Gans, Electromyography for Experimentalists. 1986, Chicago: The University of Chicago Press.

[11] Carpentier, A., J. Duchateau, and K. Hainaut, Motor unit behaviour and contractile changes during fatigue in the human first dorsal interosseus. Journal of Physiology, 2001. 534(3): p. 903-912.

[12] Basmajian, J. and C. De Luca, Muscles Alive. 1985, Baltimore, MD: Williams and Wilkins.
[13] "Biomechanics of the Hand; A Basic Research Study", Edmund Chao, Kai-Nan An, William Cooney, Ronald Linscheid (Mayo Clinic, Rochester MN), World Scientific Publishing, 1989. on page 111-113.

[14] Osu, R., Frankin, D., Kato, H., Domen, K., Yoshioka, T. Kawayo, M. Short- and long-term changes in joint cocontraction associated with motor learning as revealed from surface EMG. Journal of Neurophysiology, 2002. 88: 991-1004.

[15] Serruya, M., Hatsopoulos, N., Paninski, L., Fellows, M. Donoghue, J. Instant neural control of a movement signal. Nature, 2002. 416: 141-2

[16] Schwartz, A., Taylor, D., Tillery, S. Extraction algorithms for cotrical control of arm prosthetics. Current Opinion in Neurobiology, 2001. 11: 701-708,

[17] Gribble, P., Mullin, L., Cothros, N., Mattar, A. A role for cocontraction in arm movement accuracy. Journal of Neurophysiology, 2003. 89: 2396 - 2405.

[18] Matsuoka, Y., Howe, R. Hand Stiffness Adaptation in Positining Task with Varying Accurary Requirements. Submitted. 